\title{
Advanced Perceptual User Interfaces: Applications for Disabled and Elderly People
}

\author{
Francisco J. Perales López \\ Computer Graphics, Vision and Antificial Inteligence Group, \\ Mathematics and Computer Science Department, UIB \\ Crta. Valldemossa Km 7.5, \\ 07122 Palma de Mallorca, Spain \\ Paco.perales@uib.es
}

\begin{abstract}
The research of new human-computer interfaces has become a growing field in computer science, which aims to attain the development of more natural, intuitive, unobtrusive and efficient interfaces. This objective has come up with the concept of Perceptual User Interfaces (PUIs) that are turning out to be very popular as they seek to make the user interface more natural and compelling by taking advantage of the ways in which people naturally interact with each other and with the world. PUIs can use speech and sound recognition and generation, computer vision, graphical animation and visualization, language understanding, touch-based sensing and feedback (haptics), learning, user modeling and dialog management.
\end{abstract}

Keywords: human-computer interfaces, computer vision thecniques, natural interaction, perceptual user interfaces.

\section{Introduction}

These new interfaces can be used in different scenarios (cars, domotic houses...), but a more important issue, are the systems' potential users. PUIs offer assistive technology for people with physical disabilities, which can help them to lead more independent lives and to any kind of audience they contribute to new and more powerful interaction experiences.

Of all the communication channels through where interface information can travel, computer vision provides a lot of information that can be used for detection and recognition of human's actions and gestures, which can be analyzed and applied to interaction purposes.

When sitting in front of a computer and with the use of webcams, very common devices nowadays, heads and faces can be presumed to be visible. Therefore, system's based in head or face feature detection and tracking, and face gesture or expression recognition can become very effective human-computer interfaces. Of course, difficulties can arise from in-plane (tilted head, upside down) and out-of-plane (frontal view, side view) rotations of the head, facial hair, glasses, lighting variations and cluttered background Besides, when using standard USB webcams, the provided image resolution is very poor and it has to be taken in account. 
Different approaches have been used for non invasive face/head-based interfaces. For the control of the position some systems analyze facial cues such as color distributions, head geometry or motion. Another works track facial features or gaze including infrared lighting. To simulate the user's events it is possible to use facial gesture recognition. In this paper we consider as facial gestures the atomic facial feature motions such as eye blinking, winks or mouth's opening. Other systems contemplate the head gesture recognition that implies overall head motions or facial expression recognition that combines changes of the mentioned facial features to express an emotion

In this short course, we present an introduction to the main ideas and recent works of non invasive HCI, VBI and PUI in particular oriented to e-Accessibility for disable people. The main work done recently by Computer Graphics \& Vision Group and Artificial Intelligence from Computer and Mathematics Department at UIB will be presented. Some videos will be presented in a practical way and real demos using testing software will be used.

\subsection{Objectives, Intended Audience and Planning}

The main objectives are to show the new advances and techniques in HCI in particular VBI and their special applications to elderly users and disable people. The UE is very interested in the FP7 research program to improve the quality of live for all elderly persons and increase the social relations between older people. So the new paradigms of HCI will be a key point of research in this near future.

This area includes a multidisciplinary research collaborations son the potential users of this short course are very broad. From technician in electronics and computer science to physiologist and social career are potential professional that could be interested in this topics.

The course could be adapted to the level of audience and the time needed to explain the concepts, but four hours sessions are adequate in general. The talks are organized in one hour session. Finally one practical session could be prepared using SINA and Coldiesis software. 